\title{
Coded excitation with Nonlinear Frequency Modulation Carrier in Ultrasound Imaging System
}

\author{
Zeng FAN ${ }^{1}$, Hongying MENG ${ }^{1}$ \\ ${ }^{1}$ Department of Electronic and Electrical Engineering. Brunel University London, , London, UK \\ Zeng.Fan@bruenl.ac.uk \\ Hongying.Meng@brunel.ac.uk
}

\begin{abstract}
Since signal-to-noise ratio and axial resolution are effective decision parameters in ultrasound imaging and ultrasonic testing system, we combined nonlinear frequency modulation and Barker code sequence to improve these parameters, thereby improving the effect and performance in the ultrasound imaging and ultrasonic testing system. The theoretical study on the signal generation and decoding has been presented and the cyst phantom simulation has been carried out. The theoretical analysis shows that the new code method can improve $8.46 \mathrm{~dB}$ in Contrast ratio compared to simple pulse signal. The simulation produced the similar results.
\end{abstract}

Keywords: pulse compression; NLFM; axial resolution; SNR;

\section{Introduction}

Ultrasound is widely used in imaging and nondestructive testing (NDT) system due to its physical property [1]. In order to improve the quality of ultrasound imaging and ultrasonic testing, a very effective method which is increasing the signal to noise ratio (SNR) and axial resolution need to be invested. However, due to the high bandwidth of sidelobe, the ultrasonic testing system and ultrasonic imaging system suffer from low the axial resolution.

There are some methods such as wavelet transform to eliminate noise in ultrasonic signals. Due to the strength of filter result is bad in some application, the wavelet transform be difficult to satisfy the SNR requirements for some applications [5].

Pulse compression is frequently used in radar and medical ultrasound imaging. These techniques utilize a long sequence to achieve large main lobe energy and simultaneously to obtain low and short sidelobe. The method has two modulation method, frequency modulation and phase modulation. A linear-frequency chirp is typical frequency modulation method. The frequency of chirp signal increases (or decreases) with time. The huge difference between linear frequency method and non-linear frequency method (NLFM) is whether the frequency changing with time linearly. NLFM have the advantage that have narrower bandwidth then linear frequency method which can achieve more precise results in pulse compression technique [6]. Barker code which is one of the phase modulation methods has mostly used in Radar for many years [7] .

Katamaneni [8] studied the axial resolution comparison between LFM and NLFM, the main lobe width in NLFM reduced that can increase the axial resolution. Wang [9] used the pseudo chirp signal as carrier signal to modulate the Barker-Golay code. The ultrasound imaging simulations in FIELD II shown that the combined code has the ability to increase the SNR and axial resolution in ultrasound imaging. Juan [4] used the LFM to modulate the Barker code to increase the axial resolution in ultrasound imaging.

To improve the SNR and axial resolution, a NLFMBarker signal which is non-linear frequency modulated Barker code is applied in this paper. NLFM can attenuate the sidelobes without any SNR-robbing sidelobe filtering or window functions [2].

In this paper, basic concepts of NLFM-Barker code sequence are present. Section 2 gives the generation and compression method of NLFM-Barker code sequence. The theoretical study in section 3 compares the proposed method and other methods. The application in MATLAB shows in section 4 by using hybrid method.

\section{Modulation Methods}

Pulse compression techniques are widely used in radar and ultrasonic signal processing, which use reference signals to correlation with receive signals to increase SNR without changing average transmission power. There are two mainly methods concerned in this technique which are frequency modulation method and phase modulation method.

\subsection{Barker Code Sequences}

Barker code sequences is a typically phase modulation method in pulse compression technique. The advantage of Barker code sequence is the low the sidelobe level which constant equal to one. Due to the unique property, the lengths of Barker code sequence are only 2,3,4,5,7,11 and 13. Table 1 shows all the exist Barker code sequences. 
Table 1. Barker Code Sequences

\begin{tabular}{|c|c|c|c|}
\hline $\begin{array}{c}\text { Barker } \\
\text { code } \\
\text { length }\end{array}$ & Code & $\begin{array}{c}\text { Main } \\
\text { lobe } \\
\text { intensity }\end{array}$ & $\begin{array}{c}\text { Sidelobe } \\
\text { level(dB) }\end{array}$ \\
\hline 2 & $(+1,-1),(+1,+1)$ & 2 & -6 \\
\hline 3 & $+1,+1,-1$ & 3 & -9.5 \\
\hline 4 & $(+1,-1,+1,+1),(+1,-1,-1,-1)$ & 4 & -12 \\
\hline 5 & $+1,+1,+1,-1,+1$ & 5 & -14 \\
\hline 7 & $+1,+1,+1,-1,-1,+1,-1$ & 7 & -16.9 \\
\hline 11 & $+1,+1,+1,-1,-1,-1,+1,-1,-1,+1,-1$ & 11 & -20.8 \\
\hline 13 & $+1,+1,+1,+1,-1,-1,-1,+1,+1,-1,+1,-1,+1$ & 13 & -22.3 \\
\hline
\end{tabular}

\subsection{Non-Linear Frequency Modulation}

Linear frequency modulation(LFM) which has another name chirp signal is widely used in radar and sonar system. The instantaneous frequency of chirp signal linearly increases or decreases with time. Since the frequency bandwidth of chirp signal is much higher than the information bandwidth, the large system gain can be easily obtained [10]. This method is readily generated and can be processed by matched filter. However, the autocorrelation function of LFM waveform exhibits a sine function shape and the sidelobe structure has the same problem that can reduce axial resolution.

The common methods to reduce the sidelobe of compression output are accomplished by linear filtering or by applying window functions. the sidelobes can be reduced by this additional filter. However, since the cumulative filtering is no longer precisely matched to the signal, it will reduce output SNR by 1-2 dB.

The frequency ramp of nonlinear frequency modulation (NLFM) is non-linear. This method is no attenuation of the signal. In this paper, NLFM signal synthesis method based on the Zak transform are applied.

Here, the carrier frequency of the signal changes nonlinearly with time. The NLFM $s(t)$ could be expressed as [3]

$$
s(t)=\operatorname{Arect}\left[\frac{t-\frac{t_{i}}{2}}{t_{i}}\right] \exp (j 2 \pi \theta(t))
$$

Where $\mathrm{A}$ is the amplitude of the signal, $t_{i}$ is the signal duration time and $\theta(t)$ is the angle modulation function. To find the relationship between time and the instantaneous signal frequency, the sub-pulse number and the number of the frequency component in the sub-pulse number in the sub-pulse train model should be found. To simplify the problem, the most common way is to set the rank of norm equal to 2 .

When the rank of norm equal to 2 :

$$
\theta(t)=k-\sqrt{\left[k^{2}-(t-k)^{2}\right]}
$$

And

$$
f(t)=\frac{t-k}{\sqrt{k^{2}-(t-k)^{2}}}
$$

The instantaneous frequency of NLFM can be described by (3), when rank of norm equal to 2 . Figure 1 shows the instantaneous frequency of NLFM signal. The linear instantaneous frequency change interval $\Delta_{1}$ and instantaneous frequency change interval $\Delta_{2}$ are the main lobe width and the autocorrelation function of the NLFM signal side lobe level separately. The $\Delta_{1}$ can be described in (4).

$$
\Delta_{1}=\frac{2 \Delta_{2} \sqrt{\Delta^{2}+4}}{\Delta^{2}+4}
$$

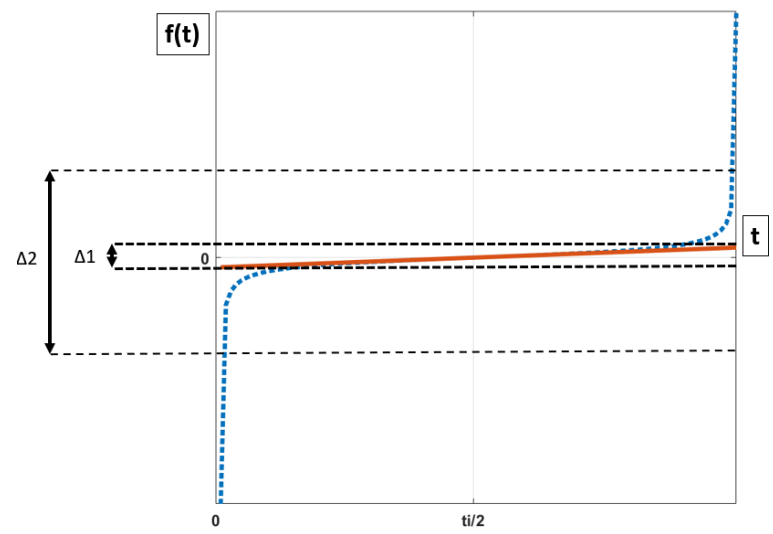

Figure 1. Example of instantaneous frquency of NLFM signal

\subsection{NLFM-Barker Hybrid method}

Due to the property of Barker code and NLFM, the hybrid method can combine the advantages of these two methods. The Barker code can be express as a convolution of carrier signal with an oversampled Barker code sequence [4]. The NLFM-Barker signal can be expressed as

$$
\mathrm{c}(t)=s(t) * \mathrm{~b}(n)
$$

Where $\mathrm{b}(n)$ is oversampled Barker sequence, $s(t)$ is carrier signal and $\mathrm{c}(t)$ is the NLFM-Barker signal. Figure 2 shows the generation and compression of NLFM-Barker code signal. 


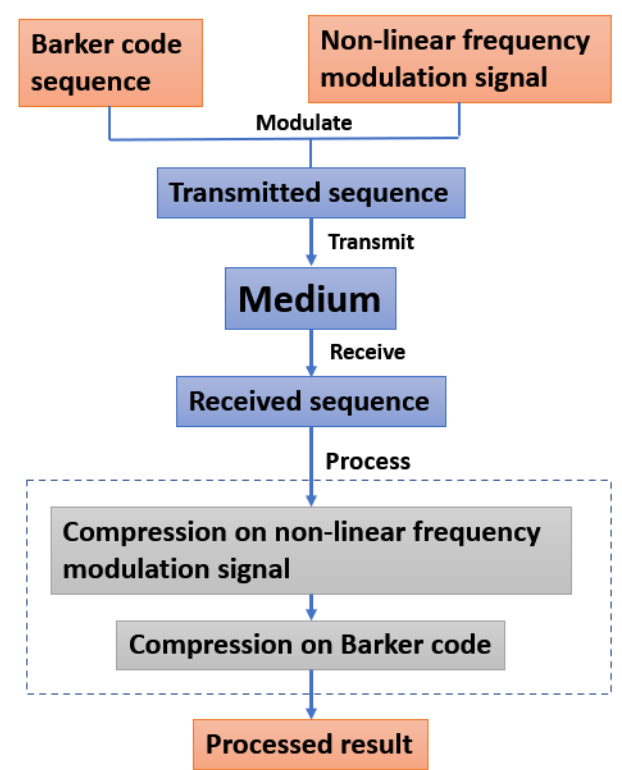

Figure 2. Generation and compression of NLFM-Barker code signal

The NLFM-Barker code signal is generated by the convolution Barker code sequence with NLFM signal. The hybrid transmitted sequence transmits in medium and the received signal will be processed by NLFM matched filter and Barker code matched filter. Figure 3 shows an example of compressed NLFM-Barker result. The input signal is NLFM signal modulation 7-bit Barker code signal. The matched filters of the hybrid signal are used to achieve the result. It can be easily seen that the pulse width of main lobe and side lobes are very narrow.

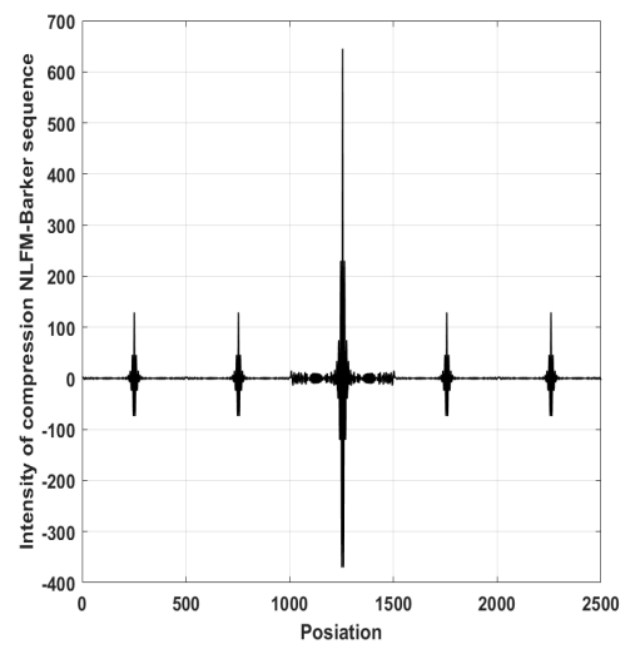

Figure 3. Echo signal of NLFM-Barker code after pulse compression.

\section{Theoretical Analysis}

In order to evaluate the performance of NLFM-Barker code, the theoretical study on axial resolution compared to other methods should be tested. The important indicator of axial resolution is the width of compressed pulse. Figure 4 compared the compression results of 7-bit Barker code sequence, LFM, NLFM and NLFM-Barker code sequence. The autocorrelation waveform of applied code method and other methods are normalized. The width of main lobe is very narrow. Figure 5 shows the zoom in of main lobe in Figure 4. Due to the normalization, the main lobe amplitude of NLFM-Barker code is the same as others, but the main lobe width of the correlation pattern has reduced compared to other code methods. The narrower lobe width can achieve more accurate results in pulse compression technique.

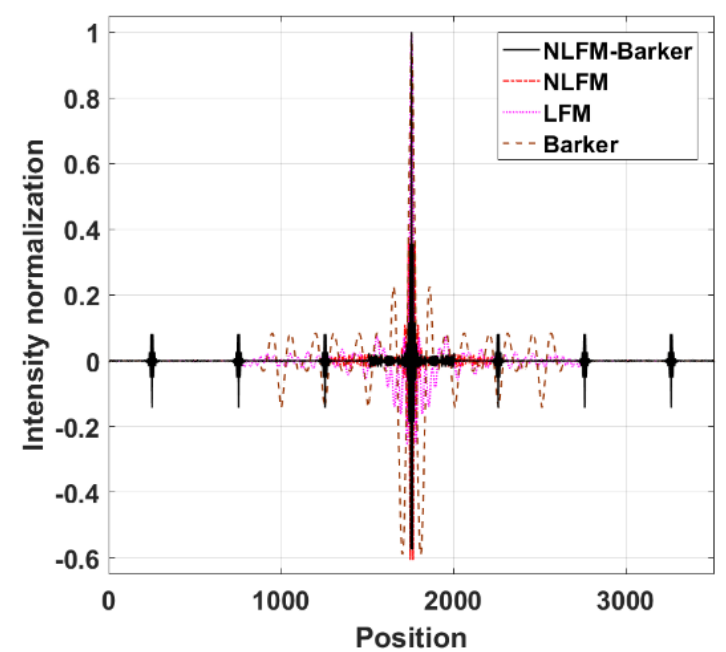

Figure 4. Autocorrelation function of 7-bit NLFM-Barker, NLFM, LFM and 7-bit Barker code sequence waveforms.

As the main lobe width is very small, the sensitivity of discrimination is high. The nearby target in the range between main lobe width can be easily distinguished.

The sidelobe level of the NLFM-Barker sequence is the same as Barker code sequence, but the sidelobe width of applied method is much smaller than Barker code sequence. Since the applied method has the property that reduce width of main lobe and side lobes, the axial resolution can be increased without using window function and other methods. This method avoids the reduce SNR of echo signal. Due to this advantage, the NLFM-Barker code sequence is considered in the ultrasound imaging area. 


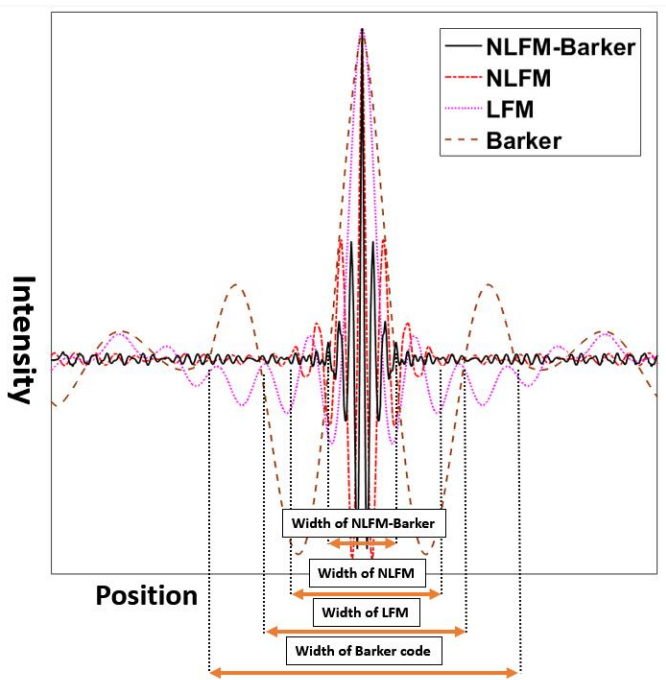

Figure 5. Zoom in of the main lobe and width of NLFMBarker and other code methods.

\section{Simulation}

The theoretical study in section 3 indicated that the NLFM-Barker code has the best performance in axial resolution than other methods. This proposed method can be used in ultrasound imaging. The phantom simulations in MATLAB have been setup in this section by using FIELD II toolbox. The linear array transducer with 192 elements which are $5 \mathrm{~mm}$ height, $440 \mathrm{um}$ width and $5 \mathrm{~mm}$ kerf. The transmit and receive focus are fixed. There are 50 scan lines in this simulation model. The center frequency of the transducer is $3.6 \mathrm{MHz}$, and the sampling frequency is 100 MHz.

The cyst phantom which consisted of 10000 points is designed in MATLAB. These points were randomly distributed with two $5 \times 5 \times 5 \mathrm{~mm}$ cubes. Figure 6 and figure 7 show the cyst phantom images obtained by simple pulse and NLFM-Barker code sequence.

Contrast ratio (CR) can intuitively contrast the performance of the applied method and simple pulse. CR and expressed as:

$$
C R=20 \log _{10} \frac{\mu_{\text {in }}}{\mu_{\text {out }}}
$$

Where $\mu_{\text {in }}$ is the mean intensity of inside the cyst and $\mu_{\text {out }}$ is the mean intensity of outside the cyst. Table 2 shows the comparison of contrast ratio of different methods.

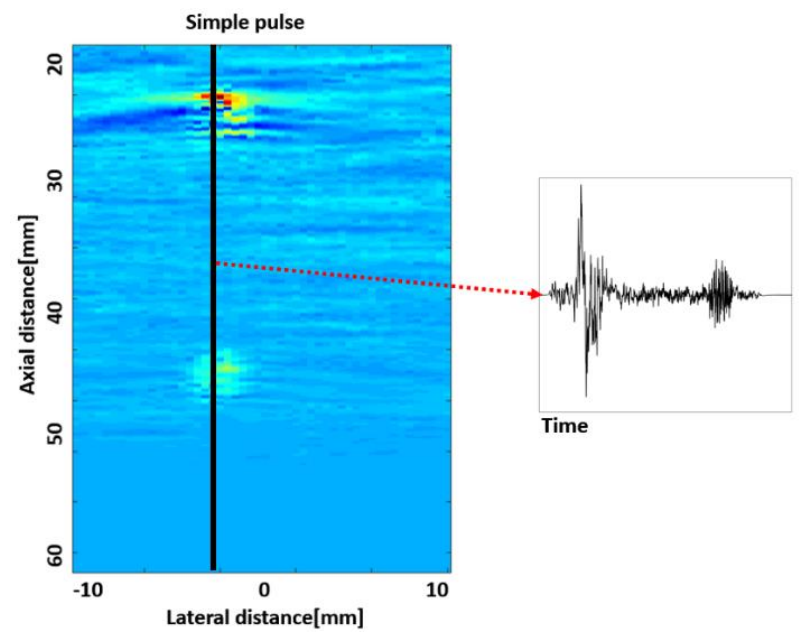

Figure 6. The simulated cyst phantom by using simple pulse and the scan line.

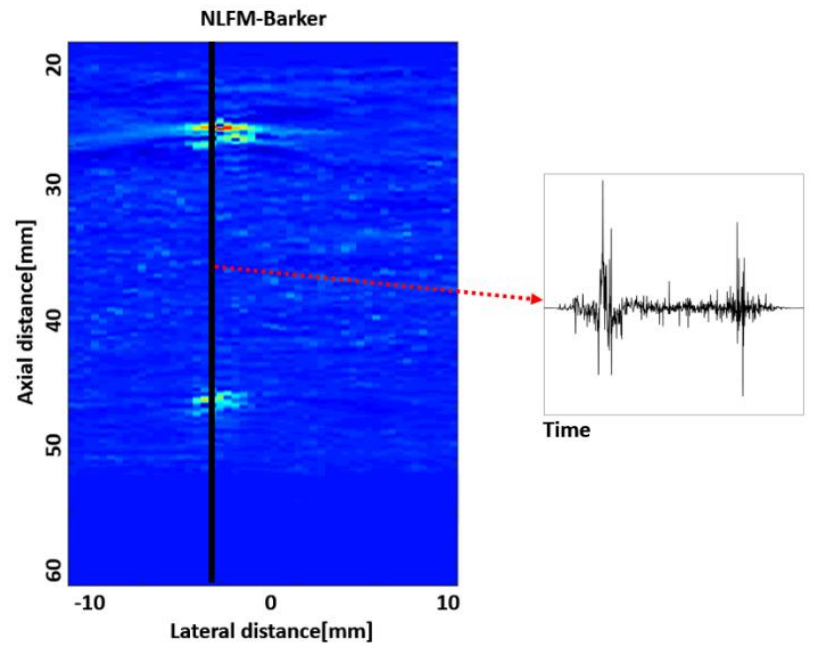

Figure 7. The simulated cyst phantom by using NLFMBarker code sequence and the scan line.

Table 2. CR of Different Code Methods

\begin{tabular}{|l|c|c|c|}
\hline Code method & $\begin{array}{c}\text { Main intensity of } \\
\text { inside cyst }\end{array}$ & $\begin{array}{c}\text { Main intensity of } \\
\text { outside cyst }\end{array}$ & $\mathrm{CR}(\mathrm{dB})$ \\
\hline Simple pulse & 6.083 & 0.743 & 18.26 \\
\hline Barker code & 13.8 & 1.101 & 21.96 \\
\hline NLFM-Barker & 31.195 & 1.438 & 26.72 \\
\hline
\end{tabular}

The CR of NLFM-Barker method is $26.73 \mathrm{~dB}$ which is higher than the simple pulse. The scan lines in figure 6 and 
7 shows that the duration time of NLFM-Barker method is smaller than the simple pulse, that can increase the axial resolution of ultrasound imaging. The performance of NLFM-Barker sequence in cyst phantom simulation agreed with the results of theoretical studies.

Due to the complicated of NLFM method, there are various of the NLFM-Barker code sequences. But the property of NLFM has the ability to reduce the width of main lobe and side lobes. All the NLFM-Barker code sequences can increase the axial resolution than other methods without losing SNR.

\section{Conclusion}

This paper used the NLFM-Barker signal as the excitation signal in ultrasound imaging. Due to the property of the signal, the axial resolution and CR have improved than other code methods significantly. The comparison in theoretical study shows that the proposed method can reduce the width of main lobe and side lobe without losing the echo signal SNR. The applied method was used in cyst phantom simulation. The simulation result in MATLAB agrees with the theoretical study. The applied signal has good performance in increasing axial resolution.

\section{Acknowledgment}

We would like to thank for the support of TWI Ltd., UK. The work was enabled through, and partially undertaken at, the National Structural Integrity Research Centre (NSIRC), a postgraduate engineering facility for industryled research into structural integrity established and managed by TWI through a network of both national and international universities.

\section{References}

[1] Z. Fan, J. Rudlin, G. Asfis and H. Meng, "Convolution of Barker and Golay Codes for Low Voltage Ultrasonic Testing", Technologies, vol. 7, no. 4, p. 72, 2019.

[2] A. Doerry, Generating nonlinear FM chirp waveforms for radar. Washington, D.C.: United States. Dept. of Energy, 2006.

[3] C. Leśnik, "Nonlinear Frequency Modulated Signal Design", Acta Physica Polonica A, vol. 116, no. 3, pp. 351-354, 2009.

[4] J. Fu, G. Wei, Q. Huang, F. Ji and Y. Feng, "Barker coded excitation with linear frequency modulated carrier for ultrasonic imaging", Biomedical Signal Processing and Control, vol. 13, pp. 306-312, 2014.

[5] Z. Zhou et al., "Application of wavelet filtering and Barker-coded pulse compression hybrid method to air-coupled ultrasonic testing", Nondestructive Testing and Evaluation, vol. 29, no. 4, pp. 297-314, 2014.

[6] I. Shahrezaei, M. Kazerooni and M. Fallah, "A Total quality assessment solution for synthetic aperture radar NLFM waveform generation and evaluation in a complex random", International Journal on Smart Sensing and Intelligent Systems, vol. 10, no. 1, pp. 174-198, 2017.

[7] R. Chiao and Xiaohui Hao, "Coded excitation for diagnostic ultrasound: a system developer's perspective", IEEE Transactions on Ultrasonics, Ferroelectrics and Frequency Control, vol. 52, no. 2, pp. 160-170, 2005.
[8] K. Sridevi, "Mainlobe width reduction using linear and nonlinear frequency modulation", in 2009 International Conference on Advances in Recent Technologies in Communication and Computing, IEEE,pp. 918--920, 2009.

[9] M. Wang, C. Shan and Z. Shi, "Pseudo Chirp-Barker-Golay coded excitation in ultrasound imaging", in 2018 Chinese Control And Decision Conference (CCDC), IEEE, 2018, pp. 4035--4039.

[10] I. Vizitiu, "Sidelobe Reduction in the Pulse-Compression Radar Using Synthesis of NLFM Laws", International Journal of Antennas and Propagation, vol. 2013, pp. 1-9, 2013 